\title{
Preguntas para pensar la relación entre justicia, reformas y feminismo
}

Q Ileana Arduino

\author{
La justicia no es solo un sentimiento. Y los sentimientos no \\ son siempre justos.
}

Pero la justicia involucra sentimientos que nos mueven a lo largo de las superficies del mundo, creando ondas en los contornos individuales de nuestras vidas. A dónde vamos con estos sentimientos, queda como una pregunta abierta.

Sara Ahmed, 2017: 304.

\section{Introducción}

Las demandas feministas que se dirigen bastante concentradamente hacia los sistemas de justicia muchas veces quedan atrapadas por comprensibles exigencias de celeridad en producir respuestas, pero solemos no detenernos en la indagación acerca de qué entendemos por justicia. Cuando se trata de recursos al poder punitivo, debemos lidiar además con el peso de acudir al núcleo duro de la configuración patriarcal del Estado porque en el mismo paso que clamamos castigo, habilitamos un juego institucional que permite confundir esa promesa con protección, sin reparar en las nuevas cargas que se imponen para obtener ese reconocimiento recortado a la condición de "víctimas" (Arduino, 2018).

Más coyunturalmente, desde hace unos meses, se colocó en el centro de la escena política un debate amplio sobre reforma judicial que derivó en un proyecto que, salvo por la inclusión ornamental de dos normas que consideran la cuestión de género, no ha receptado ninguna de las múltiples inquietudes construidas al calor del activismo feminista de los últimos años.

Incluso metodológicamente, se trata de una reforma diseñada en despachos oficiales, sin procesos de consulta -el proceso posterior quedó en manos de "notables"-que, sin cambios relevantes, se concentra en la expansión de la organización verticalizada y estanca que nos rige hoy y que constituye antes que una oportunidad para el acceso, la reafirmación de los mismos déficits de siempre (Binder, 2020). Ni las críticas ni las exaltaciones a ese proyecto son el objetivo de este artículo porque nada útil para pensar la relación sobre feminismos y justicia puede encontrarse allí. 
Sin embargo, sigue estando vacante el abordaje de problemas concretos que debieran llegar a la mesa de discusiones acerca de cómo construir una justicia con capacidad de responder a las interpelaciones feministas, pero también volver sobre cómo hemos construido las demandas mismas para comprender cuánto de lo estrecho de nuestra imaginación acerca de cómo resolverlo se cifra en la forma en que definimos nuestros problemas (Pitch, 2003 Trebisacce y Varela, 2020).

Habitualmente sucede que se asumen reflexiones en abstracto, sobre modelos teóricos o experiencias institucionales que, desde que existen, han ignorado o mostrado capacidad institucional para expulsar las demandas de justicia feminista y, cuando se las ha atendido, han constituido más bien un vector para la expansión de estructuras punitivas y de control social sesgadas racial y económicamente, producidas al mismo tiempo que retrocedían las política sociales (Kim, 2018), con resultados paupérrimos cuando se compara la promesa de la intervención y su efectivo alcance.

En esa desatención por las preguntas o problemas concretos se asume o condiciona la discusión acerca de si las respuestas deben partir de las estructuras, saberes y prácticas tal como las conocemos, con algunos "arreglos" o ajustes en los que la idea mal entendida de la perspectiva de género como apéndice y las vinculaciones entre distintos dispositivos opresivos no contaran (Guala, 2020).

Seguramente haya acuerdo en la proclama de objetivos genéricos - ¿quién no quiere el cese de la impunidad y efectividad en las respuestas? - pero ambas expresiones podrían significar un sinnúmero de cosas, y proyectos basados en consideraciones de ese tipo, sin dimensión real de los problemas, así como de su heterogeneidad, presentan alrededor consensos tan genéricos como ineficaces.

Por eso insisto en la necesidad de repensar mucho las preguntas de las cuales partimos. ¿Cómo hacer para que la justicia atienda estas demandas? Suele ser una pregunta habitual. Esa pregunta asume que el sistema tal como existe, como fue desplegándose a lo largo de la historia, es una herramienta que debería intervenir, que un poco de formación y sensibilización harán la diferencia, que alcanza con composiciones más diversificadas de los estamentos profesionales. ¿Estamos realmente de acuerdo en eso? Algunas más a modo de ejemplo: ¿cómo asegurar penas eficaces para evitar la impunidad de las violencias? Pregunto: ¿no se naturaliza allí sin siquiera problematizar un poco la relación entre pena y no impunidad? ¿Cómo garantizar acceso efectivo? ¿A qué llamamos justicia frente a las violencias? ¿Qué ofrece de transformador un sistema centrado en el castigo? ¿Hay lugar para la reparación? ¿Puede haber tutela judicial sin respeto a las autonomías?

Quisiera reivindicar aquí la importancia de construir nuevas preguntas para, al mismo tiempo que pensamos cómo debería ser la intervención, podamos aproximarnos críticamente a lo que se nos suele proponer como respuestas jurídico-judiciales ante las violencias sexo-genéricas y escoger al menos dos para ensayar posibles en las que entiendo necesario sumergirnos para romper con las formas habituales de pensar los problemas que nos acucian en este campo.

\section{¿Puede gestionarse una conflictividad tan heterogénea como la que definimos como violencias de género bajo la idea de una única forma de proceso?}

Las imposibilidades de respuestas distintas al enjuiciamiento penal suelen reivindicarse como un compromiso con las demandas de eficacia frente a las violencias de género. De hecho, en el marco del Sistema Interamericano de Derechos Humanos, 
el organismo que se ocupa de vigilar la aplicación de la Convención de Belem Do Pará (OEA, 1994) integrado por un comité de expertas, celebra desde 2012 que los países restrinjan el uso de salidas alternativas al juicio oral.

Sin embargo, la mayoría de los sistemas de justicia, ni siquiera procesa los casos que ingresan por violencias de género en un número relevante. El problema, en mi opinión, es que asiste razón a quienes señalan que esas salidas alternativas no se aplican pensando en la gestión del conflicto sino en la descongestión de la carga de trabajo y en la subestimación cultural e ideológica acerca de la relevancia que tienen estos conflictos.

Ahora bien, puede compartirse este diagnóstico, pero de ahí a concluir que la respuesta adecuada es la conducción de todos los casos en que se denuncian conflictos de estas características hasta la etapa de juicio supone que, para ser una opción preferente, tenemos sistemas que son capaces de gestionarlos y someterlos efectivamente a juicio. No es eso lo que sucede conforme la magra información empírica disponible. ${ }^{1}$ Sencillamente ocurre que los casos quedan en el camino, son archivados, muchas veces los acusados no son siquiera llamados a declarar (DGPG/MPF, 2018) y, de manera ridículamente excepcional, llegan a ser enjuiciados.

Pero, aún imaginando que existiera tal sistema que sin afectar derechos y garantías de todas las personas involucradas pudiera llevar con razonable éxito todos los casos a juicio, seguiríamos sin poder responder a la diversidad de intereses que las mujeres, en su mayoría cismujeres según los registros disponibles, expresan cuando se presentan a denunciar hechos de violencia, no necesariamente alineados con la aplicación de un castigo en un juicio penal (CEJA/INECIP, 2020).

En este sentido, la gestión de los conflictos basados en razones o contextos de violencia sexo-genérica en el ámbito del sistema de justicia no debería pensarse desconectado de las mejores condiciones de aseguramiento de la autonomía de las personas. Por el contrario, tanto los sistemas que imponen restricciones a distintas salidas que privilegien otras respuestas - composición, reparación - como aquellos que eludan el juzgamiento de casos cuando las víctimas reclaman ser oídas en juicio comprometen el respeto de la autonomía. Hacerlo en nombre de la protección de sus derechos no hace diferencias sustantivas en este punto.

Claro que pensar en esta clave, centrada en gestionar conflictos considerando la escucha conducente como parte de la respuesta integral que se pregona, pone en tensión las formas, los procedimientos, los mecanismos existentes. De eso se trata, de pensar a través de los conflictos concretos cuáles son las mejores condiciones de gestión. En un campo tan diverso y heterogéneo, renunciar a la diversidad de salidas $\mathrm{u}$ opciones como principio implica obturar existencias, intereses y necesidades que son tan diversos como las personas que los portan.

Los sistemas de tutela que constriñen el poder de decisión lesionan la autonomía y terminan por ser funcionales a las exigencias modélicas que también existen en el campo del reconocimiento de quienes son o no víctimas. La noción de audiencia (Christie, 2004) entendida como la necesidad de pensar dispositivos que saquen del silencio impuesto conflictos como las violencias en el ámbito privado, el tipo de escucha y las respuestas ante esas rupturas de silencios socialmente convalidados durante tanto tiempo reclaman una nueva política de la intervención en la gestión de los conflictos que no obture las posibilidades al atolondrarse en extender los mismos edificios conceptuales, las mismas herramientas procedimentales que se usaron en los tiempos en que se daba estructuralmente la espalda a estas violencias.

1 No voy a dedicarme a ese análisis de datos aquí, lo he hecho en otras oportunidades (Arduino, 2018b; 2020). 


\section{Llegar como víctimas, enjuiciadas como sospechosas: ¿acomodar las formas de conocimiento o pensar unas completamente nuevas?}

Por último, quisiera apuntar algunas cuestiones en torno a los modos de indagación y reconstrucción de los hechos en el campo del enjuiciamiento penal. Las prácticas dirigidas a probar ofrecen quizás una de las oportunidades más brutales para que la intervención judicial se vuelva un eslabón más de la experiencia victimizante, a fuerza de la reafirmación de estereotipos que minan desde el modo de presentar los hechos hasta las formas de valorarlos y construir argumentaciones en las decisiones o sentencias.

Los juicios sobre violencia sexista -típicamente los juicios en los que se debate acerca de la ocurrencia o no de hechos de abuso sexual como las violaciones- podrían definirse como juicios a los que se llega tras sostener cuesta arriba disputas en torno a la credibilidad de las víctimas de un modo no reconocible en el tratamiento a otras víctimas.

Así, con demasiada frecuencia, quienes llegan en calidad de denunciantes encuentran que, como resultado de la experiencia de judicialización y sus dinámicas, terminan resituadas en posición de sospechosas. En esa operación cumple un rol fundamental el modo de construir las preguntas e indagar en torno a la existencia o no de consentimiento, con peso específico en este tipo de delitos en los que la intervención jurisdiccional, lejos de esclarecer, suele acoplarse con los guiones interpretativos socialmente vigentes que dictan que lo central es evaluar cuán competente fue quien alega ser víctima en desplegar conductas de evitación del hecho, colocando en ellas la responsabilidad de evitar en base a sus propias conductas, la ocurrencia de hechos que materialmente son cometidos por otro (Kennedy, 2016).

Esos guiones ancestrales establecen que "algunas mujeres "saben" como cuidarse y otras no. Las mujeres competentes - desde la perspectiva estereotípica habitualsaben lidiar con el abuso en tres niveles. Primero, minimizan su probabilidad evitando comportamientos que podrían provocarlo, en especial "actuar como una víctima" y conducirse de una manera que "atraiga atención". Segundo, saben cómo responder en la etapa inicial de un guion de potencial abuso para orientar el curso de los acontecimientos en otra dirección. Saben cómo dejar pasar las bromas subidas de tono, pero comunican de modo claro y firme cuáles son sus límites y cuándo un hombre "se pasó de la raya". Tercero, saben reaccionar directa o indirectamente cuando está teniendo lugar un abuso, para así salvarse de un daño más grave. Abandonan al marido abusador, chillan a voz de cuello para atemorizar al violador, gritan para poner en evidencia al compañero de oficina abusador (...). Desde esta perspectiva, numerosos abusos se explican por la incompetencia de la víctima (...). En realidad, no cuentan como abusos, porque la víctima podría haberlos evitado si hubiera sabido cuidarse" (Kennedy, 2016: 33) y se replican en la instancia judicial que en lugar de erradicar prejuicios termina por perpetuarlos.

Revisar los postulados teóricos, las construcciones jurisprudenciales en torno a qué preguntas son clave para reconstruir los hechos, qué elementos probatorios son admisibles o no para discutir estos hechos y cómo se construyen las decisiones judiciales - en base a qué criterios y con qué valoraciones-constituye quizás uno de los ámbitos más diagnosticados cuando se trata de identificar expresiones judiciales de discriminación sexista y, al mismo tiempo, uno de los más desatendidos por el conocimiento jurídico que insiste en el desarrollo pretendidamente abstracto de teorías y formas de construcción del saber que deliberadamente ignoran los aportes de los feminismos sí como de otros recursos epistemológicos desarrollados desde la preocupación genuina de que el campo de la disputa judicial sea, antes que una instancia de gestión de las asimetrías, una oportunidad para la perpetuación de jerarquías. 
Este es quizás uno de los espacios donde hace falta profundizar porque, además, el fetiche pedagógico y normativo conduce muchas veces las cosas por el camino de las simplificaciones traficadas en cursos de capacitación y tips de "perspectiva" sin asumir que se trata de un problema mucho más profundo acerca de cómo conocemos, qué subjetividades reconocemos y qué testimonios resultan audibles, entre otras cuestiones (Viturro, 2007).

Implica también tomarse muy en serio la liviandad y velocidad con que muchas demandas heterogéneas, una vez encorsetadas normativamente como "violencias" encuentran como respuesta invariante la captura en nuevos delitos. Eso, sin desatender que también forma parte de las disputas el modo en que se dispone la ley como dispositivo de reconocimiento porque "Sería sin duda un error decir que todas las formas de reconocimiento son modos fugitivos de regulación y signos de falta de libertad. Tenemos que luchar por ellos en el nivel de la ley y de las políticas, aunque también tenemos que luchar contra la posibilidad de ser totalizados por ellas" (Butler y Athanasiou, 2017: 108).

\section{Para no concluir}

Este texto tiene solo la pretensión de proponer otras preguntas para un campo de discusión en el que los términos del debate suelen estar condicionados por estructuras legales e institucionales anquilosadas e introyectadas como si fueran vitales para encarar cualquier principio de solución. Las instituciones punitivas en particular gozan de esa reivindicación constante aún a cuenta de su persistente y acreditado fracaso frente a las más diversas formas de violencia.

Una primera propuesta para continuar estas conversaciones es trabajar en la identificación de elementos comunes y compartidos entre punitivismo y patriarcado (Lecumberri y Restrepo, 2016), preguntarnos cuánto de los recursos con que pensamos las respuestas dirigidas a neutralizar violencias sexo-genéricas es más funcional al mantenimiento de dispositivos opresivos que, lejos de desarticularse, confluyen y se refuerzan en las instituciones punitivas.

Hay una tensión que se plantea entre la lucha contra la impunidad selectiva de los sistemas de justicia hacia las demandas contra estas violencias y la posibilidad constante de la captura instrumental de esas demandas. Para decirlo con Tamar Pitch

(...) es fácil entender que el carácter radical de la demandas actuales es el producto (al menos) de cien años de luchas a través del derecho (...) el conjunto de temas y problemas planteados por el pensamiento feminista sobre el derecho y los derechos se puede formular con la siguiente cuestión: ¿el contrato constitutivo que se invoca como legitimación del orden moderno puede ser extendido a nuevos contratantes, o bien el irrumpir en escena de esos nuevos participantes lucha contra la injusticia y por el cambio implica necesariamente y al mismo tiempo tanto pensar un contrato radicalmente diverso? (Pitch, 2003b: 22).

Hacia adelante, ojalá encontremos, antes que mágicas respuestas, mejores preguntas concretas que empiecen también a dejar de dar por sentado que para construir justicia feminista alcanza con hacer reformas judiciales. 


\section{Q Bibliografía}

》 Ahmed, S. (2017 [2014]). La política cultural de las emociones. México, Universidad Autónoma de México, Centro de Investigaciones y Estudios de Género.

»Arduino, I. (2018). Entre la victimización opresiva y la justicia emancipatoria: articulación entre feminismo y justicia penal. En Nijensohn M. (comp.). Los feminismos ante el neoliberalismo, pp. 51-62. Buenos Aires, La Cebra/Latfem.

"-------. (2018b). Justicia penal y género: ¿un modelo para (des)armar?. Cosecha Roja, 5 de diciembre,. Disponible en: http://cosecharoja.org/55806-2/ (último acceso 30/10/2010).

"Arduino, I (2020) La respuesta penal en casos de violencia de género y el reconocimiento de la autonomía, en Kostenwein, E. (dir) El imperio de castigar. Contribuciones desde la Sociología de la justicia penal, Editores del Sur, Argentina, págs $447-464$ ( 560 pags)

" Athanasiou A. y Butler J. (2017 [2013]). Desposesión: lo performativo en lo político. Bogado, F. (trad). Buenos Aires, Eterna Cadencia.

»Binder, A. (2020). La expansión de Comodoro Py. La Nación, 10/08/2020. Disponible en: https://www.lanacion.com.ar/opinion/la-expansion-comodoropy-nid2417469 (último acceso 16/10/2020).

»CEJA/INECIP (2020). Estudio exploratorio sobre prácticas del sistema de justicia en torno a casos de violencia de género en la justicia nacional de la Ciudad de Buenos Aires: Medidas de Protección y Gestión Alternativa a los Juicios Penales. Disponible en: https://inecip.org/publicaciones/estudioexploratorio-sobre-practicas-del-sistema-de-justicia-en-torno-a-casos-deviolencia-de-genero-en-la-justicia-nacional-de-la-ciudad-de-buenos-aires/ (último acceso 16/10/20).

»Christhie, Nils (2004) una sensata cantidad de delito, Editores del Puerto, Buenos Aires, Argentina, 189 págsDGPG/MPF (2018). Informe sobre violencia en las relaciones de pareja y justicia penal: la Violencia contra las mujeres en la justicia penal, elaborado por la Dirección de políticas de Género del Ministerio Público Fiscal de la Nación, noviembre de 2018. Disponible: en: https://www. mpf.gob.ar/direcciongeneral-de-politicas-de-genero/files/2019/o4/DGPGLa-Violencia-contra-lasmujeres-en-la-justicia-penal.pdf (último acceso 8/10/2020).

"Guala, N. (2020). Feminismo antipunitivista: algunas reflexiones acerca de utilizar "las herramientas del amo". En Figueroa N. y Rovetto F. (comps.). Los feminismos frente a las violencias machistas. Conversaciones compartidas sobre nuestras prácticas cotidianas. Rosario, UNR.

»Kennedy, D. (2016). Abuso sexual y vestimenta sexy. Cómo disfrutar del erotismo sin reproducir la dominación masculina. Buenos Aires, Siglo XXI.

" Kim, M. (2018). From carceral feminism to transformative justice: Womenofcolor feminism and alternatives to incarceration. Journal of Ethnic \& Cultural Diversity in Social Work, 27:3: 219-233. Disponible en: https://www.tandfonline. com/doi/abs/10.1080/15313204.2018.1474827 (último acceso 16/10/2020).

" Lecumberri P. y Restrepo Rodríguez D. (2016). Rasgos comunes entre el poder punitivo y el poder patriarcal. Revista Colombiana de Sociología, Vol. 39 № 1, 
Bogotá, Disponible en: https://revistas.unal.edu.co/index.php/recs/article/ view/56340 (último acceso 8/10/2020).

» OEA: OEA. (1994). Convención Interamericana para prevenir, sancionar y erradicar la violencia contra la mujer, Convención de Belém do Pará [Versión Adobe Reader]. Belem do Pará, Brasi

"Pitch, T. (2003). Pitch Tamar, Responsabilidades limitadas. Actores, conflictos y justicia penal. Buenos Aires, Ad hoc.

»------. (2003b). Un derecho para dos. La construcción jurídica de género, sexo y sexualidad. Madrid, Trotta.

»Trebisacce, C. y Varela, C. (2020). Los feminismos entre la política de cifras y la experticia en violencia de género. En Daich y Varela, C. (coords.). Los feminismos en la encrucijada del punitivismo. Buenos Aires, Biblos.

»Viturro, P. (2006). Constancia. En Courtis, C. y Atienza Rodríguez, M. (pr.). Observar la ley: ensayos sobre metodología de la investigación jurídica. Madrid, Trotta. 
